\title{
El papel del orientador escolar durante la adolescencia
}

Mayra Goretti Medina Sifuentes, ${ }^{1}$ Cecilia Méndez Sánchez ${ }^{1}$

y Néstor Renedo Cámara ${ }^{1}$

\section{Introducción}

El presente trabajo es fruto de una reflexión hecha a partir de las prácticas profesionales que llevamos a cabo en el Colegio Bosques, institución educativa privada del medio urbano que cuenta con los niveles de preescolar, primaria y secundaria. En este colegio se llevó a cabo el desarrollo del Programa de Seguimiento a Alumnos (PSA), el cual consistió en brindar apoyo a la comunidad educativa cuyas áreas de acción se ubican en la evaluación psicopedagógica y psicológica y en la intervención preventiva, estimulativa y correctiva, con la finalidad de proporcionar apoyo suficiente y oportuno a los alumnos en su desempeño académico dentro de la dinámica institucional, a fin de favorecer el desarrollo de su identidad.

El objetivo del presente trabajo es abordar el papel que el orientador escolar puede realizar en las instituciones educativas mediante intervenciones psicopedagógicas con la comunidad

1 Universidad Autónoma de Aguascalientes. 
educativa y familiar a fin de favorecer el desarrollo integral del adolescente.

Este documento está enfocado en la adolescencia como objeto de estudio; se exponen los cambios que se presentan durante esta etapa de desarrollo, como alteraciones psicológicas que pudieran traer consigo repercusiones en contextos en los que interactúa el individuo así como en etapas posteriores del desarrollo.

A partir de esto se propone la figura de un orientador escolar, como aquél que funge como mediador para favorecer una educación integral en los adolescentes, haciendo un trabajo en conjunto con docentes y padres de familia; lo anterior desde el enfoque cognitivo-conductual, tomando como base los cuatro principios que rigen la orientación escolar y que justifican la acción del psicólogo: antropológico, evaluativo, del desarrollo y de intervención.

\section{Desarrollo}

\section{Radiografía del adolescente}

Adolescencia, del latín adolescere, que significa crecer (Redondo, Galdó y García, 2008). Es un periodo de transición entre la infancia y la edad adulta. Con la llegada de la pubertad surgen cambios fisiológicos, entre los que se encuentran el aumento de secreciones hormonales, la progesterona en la mujer y la testosterona en el hombre, lo cual da como resultado modificaciones somáticas. En la mujer se da la aparición de vello púbico y axilar, crecimiento de mamas, ensanchamiento de caderas y la menstruación. En el hombre se da el crecimiento del pene y los testículos, aparición de vello púbico, voz grave, eyaculación, veIlo facial y crecimiento acelerado de la estatura en ambos sexos. Estos cambios inciden directamente en el desarrollo psicológico del adolescente a nivel psicomotriz, psicosocial, afectivo y cognitivo (Coleman y Hendry, 2003).

A nivel psicomotriz se encuentran alteraciones en el esquema corporal, coordinación motora y representación espa- 
cio-temporal. A nivel psicosocial los contextos de interacción se expanden, por lo que surge una integración con el grupo de pares; estas relaciones tienden a ser en un principio homosexuales pero poco a poco se van convirtiendo en heterosexuales; por el contrario, las relaciones con el contexto familiar se van debilitando y la oposición a la autoridad se hace presente. Afectivamente, la autoimagen, el autoconcepto, la autoaceptación y la autoestima se encuentran alterados.

Cognitivamente se encuentra el egocentrismo intelectual, lo cual dificulta el pensamiento abstracto para la solución de problemas, razonamiento, formulación de hipótesis, reflexión, análisis, exploración de variables y alternativas que intervienen en un fenómeno (Álvarez, 2010).

Las alteraciones que pudieran presentarse en el adolescente traen consigo repercusiones en su desarrollo. Estas repercusiones se pueden observar en los ámbitos en los que éste interactúa, es decir, en la familia, su grupo de iguales y en las instituciones educativas (Coleman y Hendry, 2003).

Asimismo, dichos contextos fungen como factor de protección o de riesgo para desencadenar alteraciones de desarrollo durante la adolescencia. Es por ello indispensable el papel de una figura que funja como mediador en el proceso formativo institucional, siendo éste el orientador escolar. Como tal, deberá favorecer a una educación integral en los adolescentes, mediante un proceso de evaluación e intervención con todo el personal que interactúa en la institución educativa (Pérez y Rivera, 2005).

\section{Fundamento del trabajo del orientador escolar}

El trabajo psicológico del orientador escolar está fundamentado en el enfoque cognitivo-conductual, según el cual la conducta humana es aprendida. Se entiende por conducta la interacción del individuo con su medio. Dicha conducta se verá fortalecida o debilitada por sus consecuencias (Kazdin, 2000). La conducta de los individuos puede ser observable, medible y cuantificable. 
Desde esta perspectiva, no pasan desapercibidos los procesos internos como los pensamientos y las emociones, sino que son entendidos de igual manera como conductas. Los cognitivos conductuales se diferencian de los conductistas al plantear que los pensamientos son el origen y la causa de las conductas observables así como de las emociones; además, se concibe el individuo como poseedor de un sistema de procesamiento de la información, capaz de organizar, sistematizar y crear información, es decir, un ente activo en el desarrollo de su conducta (Mediavilla y Martínez, 2003).

\section{Agentes involucrados en el orientador escolar}

El orientador escolar es el profesionista de la psicología que funge como mediador en el proceso formativo, de tal manera que atiende y responde de manera efectiva a las demandas y necesidades del adolescente tanto de manera individual como grupal, trabajando en conjunto con los docentes y los padres de familia (Mediavilla y Martínez, 2002).

El profesionista deberá ser competente y eficaz tanto en el plano teórico como práctico, aplicar sus habilidades de evaluación e intervención de acuerdo con las necesidades de todos los agentes inmersos en la institución. Por otro lado, contar con actitudes de responsabilidad, innovación, liderazgo, empatía, tolerancia, respeto, honestidad, congruencia y objetividad por parte del orientador resulta ser fundamental (SEP, 2010).

\section{Áreas de acción}

"Para que el orientador alcance sus objetivos y cumpla sus funciones, habrá de tomar en cuenta los principios siguientes, los cuales fundamentan y justifican su actuar" (Mediavilla y Martínez, 2002). 
- Antropológico: conceptualizar al adolescente como un ser individual, capaz de asumir responsabilidades tanto de sí mismo como de los demás, clarificar objetivos y dirigir la atención de manera individualizada e integradora.

- Evaluativo: va dirigida a un individuo, grupo, familia y docentes, lo cual implica evaluar sus necesidades utilizando como recursos la observación, entrevista, pruebas psicométricas, culminando con el análisis de los resultados.

- Del desarrollo: dotar al adolescente de competencias de acuerdo a su etapa de desarrollo en el área psicomotriz, psicosocial, afectiva y cognitiva.

- Intervención: con base en el análisis de los resultados del proceso de evaluación, incidir en el problema mediante un proceso de intervención en distintos niveles:

- Primario: actuar sobre los factores que originan el problema, evitando el riesgo de aparición.

- Secundario: actuar sobre los problemas que ya han aparecido para eliminarlos.

- Terciario: incidir sobre los problemas para reducirlos.

Respecto al proceso de evaluación, resultan ser importantes las entrevistas y encuestas a profesores y padres de familia sobre situaciones que pudieran estar alterando la dinámica educativa y familiar. De igual manera, resulta esencial realizar registros de observación sobre profesores y alumnos dentro o fuera del salón de clase, que den cuenta de los métodos de enseñanza-aprendizaje y la dinámica entre grupo de iguales y sus profesores. Los productos derivados de la implementación de estas herramientas permitirán realizar un análisis en el que se identifiquen las demandas y necesidades y, como consecuencia, generar las intervenciones pertinentes (Nérici, 1976).

La intervención que se realice podrá darse en distintos niveles; por un lado, de manera individual en la atención de las demandas referidas por el adolescente o por los docentes y, por el otro, de manera grupal y/o colectiva mediante talleres formativos que busquen satisfacer sus necesidades y en los que se aborden aspectos de vital importancia durante esta etapa de de- 
sarrollo (SEP, 2010). Es importante hacer frente a todas las demandas que se presentan en la institución, independientemente del grado de relevancia, con la finalidad de llegar a hacer un trabajo en su mayoría preventivo.

Los temas versarían sobre procesos personales, académicos y de responsabilidad social. En los procesos personales encontramos la identidad, planificación y proyecto de vida, autocontrol, identificación y expresión de emociones, desarrollo de valores, solución de problemas, toma de decisiones, comunicación asertiva, relaciones interpersonales, trabajo en equipo, creatividad y orientación vocacional (SEP, 2010). En cuanto a los procesos académicos se encuentran los referentes al proceso enseñanza-aprendizaje, fenómenos de inadaptación y deserción escolar, hábitos de estudio y aprender a aprender. Por último, en los procesos de responsabilidad social encontramos la educación cívica y moral, educación para la salud, educación sexual, adicciones y conductas antisociales (Mediavilla y Martínez, 2002).

Es indispensable trabajar en estrecha colaboración con los profesores y padres de familia, puesto que intervenir únicamente con el adolescente sería muy limitado, debido a que éste interactúa con ambos grupos. De ahí la importancia de llevar a cabo un trabajo en conjunto para propiciar una educación integral en él (Hill, 1973). Como punto de partida, resulta importante atender a las ideas y actitudes que docentes y padres de familia tienen acerca de la adolescencia mediante cursos de capacitación sobre esta edad evolutiva. No podemos dejar de lado la atención a las demandas que tanto profesores como padres de familia presentan. De igual manera, resulta fundamental que los profesores reciban capacitaciones en cuanto a métodos de enseñanza-aprendizaje, comunicación asertiva, solución de problemas y toma de decisiones. Asimismo, los padres de familia deberán conocer estrategias para mejorar la dinámica familiar y estilos de crianza. No pueden faltar las evaluaciones durante y al término de las intervenciones con el objetivo de evaluar su eficacia y de realizar modificaciones en caso de ser necesario (Mediavilla y Martínez, 2003). 
La interacción y retroalimentación tripartita entre profesores, padres y orientador es el alma de la formación del joven, pues propicia acciones pertinentes, acertadas y conjuntas en relación con el adolescente, basadas en la observación, en la información y en la reflexión.

\section{Conclusión}

Realizar un trabajo psicológico enfocado en los adolescentes y en las personas que interactúan con éste permite generar acciones oportunas en el ámbito educativo para favorecer el desarrollo psicológico del adolescente y propiciar una transición satisfactoria hacia la vida adulta. Es necesario que las acciones del orientador escolar estén fundamentadas bajo un marco teórico de referencia que dé sustento a su actuar e incida sobre los cuatro principios que sustentan la orientación escolar, sin dejar de lado las competencias indispensables que como profesionista debe tener para satisfacer las demandas y necesidades de la institución.

Cabe señalar que las áreas de acciones propuestas para el orientador escolar no son preestablecidas o que pudieran aplicarse de igual manera en todos los contextos educativos, puesto que es necesario tomar en cuenta el contexto social, características socioeconómicas y culturales de toda la comunidad educativa y variables que enmarcan el trabajo global del orientador. Asimismo, resulta importante tomar en cuenta las características de la etapa de desarrollo en la que se encuentra nuestro individuo focal, debido a que el trabajo psicológico con niños, adolescentes, jóvenes y adultos resulta ser diferente por las características que conlleva cada etapa.

A partir de esto, se debe realizar un programa individualizado de apoyo, basado en el conocimiento personal de cada persona, fortalecido con un trabajo en conjunto con los docentes y los padres de familia, lo cual dará la posibilidad de realizar un trabajo integral para favorecer al adolescente al dotarle de herramientas, en donde pueda hacer frente a las situaciones que se presenten en sus diversos contextos de interacción. 


\section{Referencias}

Álvarez, J.M. (2010). Características del desarrollo psicológico del adolescente. Innovación y Experiencias Educativas, 28(1-11).

Coleman, J.C. y Hendry, L.B. (2003). Psicología de la adolescencia. Madrid: Morata.

Hill, G.E. (1973). Orientación escolar y vocacional. México: Pax. Kazdin, A.E. (2000). Modificación de la conducta y sus aplicaciones prácticas. México: El Manual Moderno. (Trabajo original de publicación 1996).

Lineamientos de Orientación Educativa. Dirección General de Bachillerato, 2010. Santiago de Chile, Ediciones Dolmen. Recuperado de http://www.dgb.sep.gob.mx.

Mediavilla, L.G. y Martínez, M. (2002). La orientación escolar: Fundamentos y desarrollo. Madrid: Dikynson.

Mediavilla, L.G. y Martínez, M. (2003). Orientación educativa en la familia y en la escuela. Madrid: Dikynson.

Nérici, I.G. (1976). Introducción a la orientación escolar. Brasil: Kapelusz.

Pérez, A. y Rivera, A. (2005). La orientación escolar en centros educativos. Madrid: Ministerio de Educación y Ciencias.

Redondo, C.G., Galdó, G. y García, M. (2008). Atención al adolescente. España: Universidad de Cantabria. 